\title{
Mediterranean diet intervention in rheumatoid arthritis
}

\section{J Kjeldsen-Kragh}

\section{Is the controlled clinical trial the only tool for testing the efficacy of dietary treatment?}

7 he belief that diet may influence the severity of rheumatoid arthritis (RA) is part of the folklore of the disease, and dietary treatment has been one of many unconventional remedies that have been widely used at health farms in northern Europe. Over the years, several stories about arthritic patients who improved dramatically after changing diet have appeared, not only in the lay press but also in scientific journals. Although the possible benefits of specific diets for patients with RA have been tested in a number of trials, many of the studies that showed positive results of dietary treatment have not been published as full papers in peer reviewed journals, probably because these studies lacked controls or were poorly designed and/or inadequately described.

A scientific investigation of the efficacy of dietary treatment of RA was pioneered by Swedish doctors. More than two decades ago Sköldstam et al conducted the first controlled randomised trial, in which they tested the effect of fasting and lactovegetarian diet in patients with RA. ${ }^{1}$ They reported that fasting resulted in a striking reduction in disease activity but, unfortunately, the patients deteriorated again when they started eating. Since then only a few randomised controlled trials on dietary treatment of patients with RA have been carried out. ${ }^{2-8}$ These trials are not directly comparable because of differences in the diets, and accordingly the results varied from no effect ${ }^{2}$ to good clinical response to the experimental diet. ${ }^{4}$

\section{WHY DOES DIET HELP SOME PATIENTS WITH RA?}

There has been much speculation about why some patients with RA improve by changing diet. Allergy or other adverse antibody mediated reactions towards certain foods have been suggested, ${ }^{39-13}$ but seem to have a role in only a few patients with RA. ${ }^{11}{ }^{14}$ This may also explain why the clinical results of elemental diet have been disappointing. ${ }^{11}{ }^{15-17}$ Dietary induced alterations in the profile of prostanoid precursors have also been suggested as a possible explanation, but evidence for this hypothesis has not been provided. ${ }^{18}$ A significant alteration in the gut flora has been reported to be associated with clinical improvement in patients with RA during dietary treatment. ${ }^{19} 20$ Conceivably, absorption of some microbial antigens has a role in the pathogenesis of RA and if the intestinal flora is altered owing to a changed diet this might have favourable effects on the disease activity.

\section{TRADITIONAL CRETAN DIET}

Epidemiological studies have suggested that a diet rich in fish, ${ }^{21}{ }^{22}$ olive oil, ${ }^{23} 24$ and cooked vegetables ${ }^{24}$ might have a protective effect against RA. The Mediterranean diet, particularly the traditional diet from Crete, has been regarded as particularly healthy. This diet, which is characterised by a high content of cereals, vegetables, legumes, fruits, and olive oil, has been shown to reduce the recurrence rate of new cardiac events. ${ }^{25}$ Because atheromatosis, like RA, has a strong inflammatory component, an intriguing question is whether the traditional Cretan diet might also alleviate the symptoms of RA.

"Diets rich in fish, olive oil, and cooked vegetables may protect against RA"

To answer this query, Sköldstam et al carried out a randomised controlled trial over three months in which the traditional Cretan diet was compared with an ordinary omnivorous diet in 56 patients with active RA. ${ }^{26}$ The primary efficacy variables were a composite disease activity index (DAS28), physical function using the Health Assessment Questionnaire (HAQ), a health survey of quality of life (SF-36), and the daily consumption of non-steroidal antiinflammatory drugs. In the experimental diet group they observed a significant improvement after three months in the DAS28 score, in the HAQ score, in two dimensions of the SF-36 health survey, and in six of 10 secondary efficacy variables. No significant improvements were seen in the group of omnivores. In addition, when the change during the trial was compared between groups, the patients following the traditional Cretan diet had a significantly more favourable course than the omnivores for the DAS2 8 score, the HAQ score, and for three of the secondary variables. Individually, 15 patients in the experimental diet group, compared with only six patients in the control group, showed a clinical significant improvement-that is, a decrease in the DAS28 score of $>0.6$.

Thus, it seems that the traditional Cretan diet can suppress disease activity in patients who have stable and modestly active RA. Sköldstam et al, however, are cautious about their conclusion, and emphasise that it remains to be shown if long term consumption of this diet is beneficial for patients with RA. Long term studies are indeed crucial when new treatments are evaluated for a chronic disease such as RA, but, hitherto, only two randomised controlled oneyear trials on the dietary treatment of RA have been conducted. ${ }^{4}$

PROBLEMS WITH CLINICAL TRIALS OF DIETARY TREATMENT

Numerous problems are associated with clinical trials of dietary treatment and Sköldstam et al should be acclaimed for a well planned and well conducted study. These kinds of trial are faced with other problems than the usual trials testing pharmaceutical drugs.

\section{Recruitment}

Firstly, recruitment of patients is much more difficult because the treatment requires the patients to change their lifestyle. In ordinary drug trials patients have to take a certain number of pills every day, but in trials evaluating dietary treatment patients in the experimental diet group are asked to renounce their dietary habits, usually with severe implications for the patient's social life.

\section{Compliance}

Secondly, it is difficult to monitor patient compliance. To ensure optimal compliance the patients in the study by Sköldstam et al were served either the experimental diet or the control diet during the three weeks they attended an outpatient based rehabilitation programme. In addition, the patients in the experimental diet group had six lessons from a dietician about Mediterranean food and cooking and during the remaining part of the study they could contact the dietician weekly for advice.

\section{Drop outs}

Thirdly, the number of patients who prematurely discontinue the studies of dietary treatment is usually very high in clinical trials of dietary treatment. This is not surprising because dietary habits are 
a fundamental feature of daily life. However, the number of drop outs in clinical trials always makes interpretation of the results difficult. Therefore, in dietary studies a close follow up by a qualified dietician is of utmost importance in order to ensure both an acceptable compliance and to minimise the number of patients who prematurely discontinue the study. It appears that Sköldstam et al have succeeded in this respect as only three patients in the experimental diet group left the trial, and, of these, only two seemed to leave for reasons directly related to the diet.

\section{Interpretation of results}

Fourthly, interpretation of the results is obscured by both the placebo and the nocebo effect. The "gold standard" for clinical trials is the double blind randomised clinical trial, but for obvious reasons a study investigating the efficacy of a particular diet cannot be conducted in a double blind fashion. Thus, for studies of dietary treatment one can never rule out the possibility that an improvement is mainly caused by the placebo effect. Patients enrolled in such studies are highly motivated and they are most probably not representative of patients with RA in general. Many of these patients are thrilled by the idea that the disease can be controlled by dietary intervention, and it is not unlikely that such an attitude may promote the placebo effect if they are randomly allocated to the experimental group. ${ }^{27}$ On the contrary, patients randomly allocated to a control group, continuing to follow an omnivorous diet, may be disappointed and experience the opposite of the placebo effect-namely, the nocebo effect.

\section{Funding}

Finally, funding of studies testing the efficacy of dietary manipulation is much more difficult than for drug trials because there are no commercial interests linked to the treatment.

All these circumstances probably explain why so few clinical trials of dietary treatment have been carried out during the past two decades.

\section{DOUBLE BLIND STUDIES VERSUS SINGLE ARM TRIALS}

From the study by Sköldstam et al and previous randomised trials on dietary treatment of RA it seems that at least some patients benefit clinically from dietary manipulation. Unfortunately, our knowledge is still scarce because we do not know the mechanisms behind the clinical improvement, but even more importantly, confronted with a patient we are not able to tell which particular diet is the best and if improvement can be expected or not. These are practical clinical problems that many rheumatologists face every day. Is it reasonable to believe that the controlled randomised clinical trial is a suitable tool for clarifying these questions? The methodological impediments inevitably associated with the randomised controlled clinical trial limit the conclusions to be drawn from such studies: firstly, because the included patients are highly motivated for lifestyle changes and therefore not representative of the general population of patients with RA; and secondly, because it is impossible to rule out the possibility that a beneficial effect is due to the placebo effect. In clinical trials patients are traditionally randomly allocated to one of two treatment groups in order to distinguish the "real" effect from the placebo effect, but in studies of the efficacy of lifestyle changes it is not possible to separate the "real" effect from the placebo effect.

Acknowledging the existence of the aforementioned problems, it is difficult to consider the controlled double blind clinical trial as the "gold standard" for studies examining the clinical efficacy of lifestyle changes. An alternative is the single arm trial, in which all patients interested in dietary treatment are included in one group. The single arm trial would resemble the everyday clinical setting much better, because randomisation is not a part of the ordinary consultation with a doctor. One may ask: which important questions could be answered by conducting only single arm trials? This kind of trial might be very useful for answering the essential clinical question: how can we identify diet responders? We know that only a certain percentage of patients with RA benefit from dietary manipulation and it would be of considerable practical importance to be able to predict with some degree of certainty if a particular patient would improve clinically or not. At the time of inclusion, information about a number of variables, which are presumed to be of importance for the success, should be acquired for each patient. Based on a priori presumptions sex, disease duration, disease activity, and rheumatoid factor status might be examples of such variables. At the end of the trial the success of the treatment should be scored for each patient. A multiple regression model using treatment success as the dependent variable and the baseline variables as independent variables is a powerful tool to identify which baseline variables can be used to predict diet responders.

Using this approach several other aspects of dietary treatment can also be examined. For example, if we want to examine whether the dietary intake of certain foods is associated with improvement we just need to select another set of independent variables.
In this kind of trial we do not need to be concerned about the extent to which the placebo effect is acting, and this also resembles the everyday clinical setting much more than the traditional double blind randomised clinical trial. Moreover, it will probably be easier to recruit patients because they do not have to accept randomisation. All patients will receive the experimental treatment and, consequently, a control group will be redundant. Although such single arm trials can provide us with essential information, it is important to understand that the acquired knowledge will merely be a basis for new hypotheses, which in turn need to be tested in subsequent trials. Thus, these trials combined with multiple regression analysis should not be considered as alternatives to controlled clinical trials but as a supplement to them.

\section{FINAL COMMENTS}

Sköldstam et al have shown that a traditional Cretan diet has a beneficial short term effect on RA. The authors deserve credit because they have undertaken a tremendous task by thoroughly planning and conducting the trial. They should also be acknowledged for their endeavour to carry out a follow up study after one year. However, both the workload and the methodological problems associated with controlled clinical trials discourage many clinicians from exploring the effect of dietary treatment of RA. This is probably the explanation why only two one-year controlled clinical trials have been reported so far. ${ }^{48}$ Therefore, it is important to recognise that essential clinical information can be obtained by performing single arm trials. They are easier to carry out, and although there are also methodological problems associated with this kind of trial, they can still be considered as a valuable supplement to the controlled double blind clinical trial.

Ann Rheum Dis 2003;62:193-195

\section{Authors' affiliations}

J Kieldsen-Kragh, Department of Immunology and Transfusion Medicine, Ullevaal University Hospital, Oslo, Norway

Correspondence to: Dr J Kjeldsen-Kragh, Department of Immunology and Transfusion Medicine, Ullevaal University Hospital, Kirkeveien 166, N-0407 Oslo, Norway; jens.kjeldsen-kragh@ioks.vio.no

\section{REFERENCES}

1 Sköldstam L, Larsson L, Lindström FD. Effect of fasting and lactovegetarian diet on rheumatoid arthritis. Scand J Rheumatol 1979;8:249-55

2 Panush RS, Carter RL, Katz P, Kowsari B, Longley S, Finnie S. Diet therapy for rheumatoid arthritis. Arthritis Rheum 1983;26:462-71.

3 Darlington LG, Ramsey NW, Mansfield JR. Placebo-controlled, blind study of dietary 
manipulation therapy in rheumatoid arthritis. Lancet 1986;i:236-8

4 Kjeldsen-Kragh J, Haugen $M$, Borchgrevink $C F$, Laerum E, Eek M, Mowinkel P, et al. Controlled trial of fasting and one-year vegetarian diet in rheumatoid arthritis. Lancet 1991;338:899-902.

5 Hansen GV, Nielsen L, Kluger E, Thysen M, Emmertsen $\mathrm{H}$, Stengaard-Pedersen $\mathrm{K}$, et al. Nutritional status of Danish rheumatoid arthritis patients and effects of a diet adjusted in energy intake, fish-meal, and antioxidants. Scand J Rheumatol 1996;25:325-30.

6 Nenonen MT, Helve TA, Rauma AL, Hanninen OO. Uncooked, lactobacilli-rich, vegan food and rheumatoid arthritis. $\mathrm{Br} J$ Rheumatol 1998;37:274-81.

7 Sarzi-Puttini P, Comi D, Boccassini L, Muzzupappa S, Turiel M, Panni B, et al. Diet therapy for rheumatoid arthritis. A controlled double-blind study of two different dietary regimens. Scand J Rheumatol 2000;29:302-7

8 Hafstrom I, Ringertz B, Spangberg A, von Zweigbergk L, Brannemark S, Nylander I, et al. A vegan diet free of gluten improves the signs and symptoms of rheumatoid arthritis: the effects on arthritis correlate with a reduction in antibodies to food antigens. Rheumatology (Oxford) 2001;40:1175-9.

9 Panush RS. Food induced ("allergic") arthritis: clinical and serologic studies. Rheumatol 1990;17:291-4

10 van de Laar MA, Aalbers M, Bruins FG, van Dinther-Janssen AC, van der Korst JK, Meijer CJ. Food intolerance in rheumatoid arthritis. II. Clinical and histological aspects. Ann Rheum Dis 1992;51:303-6.

11 van de Laar MA, van der Korst JK. Food intolerance in rheumatoid arthritis. I. A double blind, controlled trial of the clinical effects of elimination of milk allergens and azo dyes. Ann Rheum Dis 1992;51:298-302.

12 Kjeldsen-Kragh J, Hvatum M, Haugen M, Førre $\varnothing$, Scott $H$. Antibodies against dietary antigens in rheumatoid arthritis patients treated with fasting and a one-year vegetarian diet. Clin Exp Rheumatol 1995; 13:167-72.

13 Hafström I, Ringertz B, Gyllenhammer H, Palmblad J, Harms-Ringdahl M. Effects of fasting in disease activity, neutrophil function, fatty acid composition, and leukotriene biosynthesis in patients with rheumatoid arthritis. Arthritis Rheum 1988;31:585-92.

14 Felder M, De Blecourt AC, Wuthrich B. Food allergy in patients with rheumatoid arthritis. Clin Rheumatol 1987:6:181-4.

15 Haugen MA, Kjeldsen-Kragh J, Førre $\varnothing$. A pilot study of the effect of an elemental diet in the management of rheumatoid arthritis. Clin Exp Rheumatol 1994;12:275-9.

16 Holst-Jensen SE, Pfeiffer-Jensen M, Monsrud M, Tarp U, Buus A, Hessov I, et al. Treatmen of rheumatoid arthritis with a peptide diet: a randomized, controlled trial. Scand J Rheumatol 1998:27:329-36.

17 Kavanaghi R, Workman E, Nash P, Smith M, Hazleman BL, Hunter JO. The effects of elemental diet and subsequent food reintroduction on rheumatoid arthritis. $\mathrm{Br} J$ Rheumatol 1995:34:270-3.

18 Haugen MA, Kjeldsen-Kragh J, Bjerve KS Høstmark AT, Førre $\varnothing$. Changes in plasma phospholipid fatty acids and their relationship to disease activity in rheumatoid arthritis patients treated with a vegetarian diet. $\mathrm{Br}$ Nutr 1994;72:555-66.

19 Peltonen R, Kjeldsen-Kragh J, Haugen M, Tuominen J, Toivanen P, Førre $\varnothing$, et al. Changes of faecal flora in rheumatoid arthritis during fasting and one-year vegetarian diet. $\mathrm{Br}$ J Rheumatol 1994;33:638-43.
20 Peltonen R, Nenonen M, Helve T, Hanninen $\mathrm{O}$, Toivanen P, Eerola E. Faecal microbia flora and disease activity in rheumatoid arthritis during a vegan diet. $\mathrm{Br} J$ Rheumatol 1997; 36:64-8.

21 Shapiro JA, Koepsell TD, Voigt LF, Dugowson CE, Kestin M, Nelson JL. Diet and rheumatoid arthritis in women: a possible protective effect of fish consumption. Epidemiology 1996;7:256-63.

22 Recht L, Helin P, Rasmussen JO, Jacobsen J Lithman T, Schersten B. Hand handicap and rheumatoid arthritis in a fish-eating society (the Faroe Islands). J Intern Med 1990;227:49-55

23 Linos A, Kaklamanis E, Kontomerkos A, Koumantaki Y, Gazi S, Vaiopoulos G, et al The effect of olive oil and fish consumption on rheumatoid arthritis - a case control study. Scand J Rheumatol 1991;20:419-26.

24 Linos A, Kaklamani VG, Kaklamani E, Koumantaki Y, Giziaki E, Papazoglou S, et al. Dietary factors in relation to rheumatoid arthritis: a role for olive oil and cooked vegetables? Am J Clin Nutr 1999:70:1077-82.

25 de Lorgeril M, Renaud S, Mamelle N, Salen P, Martin JL, Monjaud I, et al. Mediterranean alpha-linolenic acid-rich diet in secondary prevention of coronary heart disease. Lancet 1994;343: 1454-9.

26 Sköldstam L, Hagfors L, Johansson G. An experimental study of a Mediterranean diet intervention for patients with rheumatoid arthritis. Ann Rheum Dis 2003:62:208-14.

27 Kjeldsen-Kragh J, Haugen M, Førre $\varnothing$, Laache H, Malt UF. Vegetarian diet for patients with rheumatoid arthritis: can the clinical effects be explained by the psychological characteristics of the patients? Br J Rheumatol 1994;33:569-75. 